IAC-14.A1.2.2

\title{
UNDERSTANDING THE EFFECTS OF SPACEFLIGHT ON HEAD-TRUNK COORDINATION DURING WALKING AND OBSTACLE AVOIDANCE
}

\author{
S. Madansingh \\ University of Houston, Department of Health and Human Performance, Houston, Texas, USA \\ simadansingh@uh.edu \\ J.J. Bloomberg, PhD \\ NASA Johnson Space Center, Neuroscience Laboratory, Houston, Texas, USA \\ jacob.j.bloomberg@nasa.gov
}

Prolonged exposure to spaceflight conditions results in a battery of physiological changes, some of which contribute to sensorimotor and neurovestibular deficits. Upon return to Earth, functional performance changes are tested using the Functional Task Test (FTT), which includes an obstacle course to observe post-flight balance and postural stability, specifically during turning. The goal of this study was to quantify changes in movement strategies during turning events by observing the latency between head-and-trunk coordinated movements. It was hypothesized that subjects experiencing neurovestibular adaptations would exhibit head-to-trunk locking ('en bloc' movement) during turning, exhibited by a decrease in latency between head and trunk movement. FTT data samples were collected from 13 ISS astronauts and 26 male 70-day head down tilt bed rest subjects, including bed rest controls (10 BRC) and bed rest exercisers (16 BRE). Samples were analyzed three times pre-exposure, immediately post-exposure (0 or 1 day post) and 2-to-3 times during recovery from the unloading environment. Two 3D inertial measurements units (XSens MTx) were attached to subjects, one on the head and one on the upper back. This study focused primarily on the yaw movements about the subject's center of rotation. Time differences (latency) between head and trunk movement were averaged across a slalom obstacle portion, consisting of three turns (approximately three $60^{\circ}$ turns). All participants were grouped as 'decreaser' or 'increaser,' relating to their change in head-to-trunk movement latency between pre- and post- environmental adaptation measures. Space flight unloading (ISS) showed a bimodal response between the 'increaser' and 'decreaser' group, while both bed rest control (BRC) and bed rest exercise (BRE) populations showed increased preference towards a 'decreaser' categorization, displaying greater head-trunk locking. It is clear that changes in movement strategies are adopted during exposure to an unloading environment. These results further the understanding of vestibular-somatosensory convergence and support the use of bed rest as an exclusionary model to better understand sensorimotor changes in space flight.

\section{INTRODUCTION}

Adaptation to the microgravity environment experienced by astronauts during long-duration space flight (6-month missions aboard the International Space Station) has a number of deleterious effects on the body, affecting mainly those physiological systems reliant on Earth's constant 1g acceleration for normal functioning. These changes include musculoskeletal deconditioning[1-3], decreased cardiovascular function[4,5], often characterized by orthostatic intolerance[6,7], and adaptive modification of sensorimotor systems leading to postural and locomotor disturbances[8-12]. Gravity is sensed by the vestibular organs, particularly the otoliths, and is detected by proprioceptive and somatosensory systems due to gravitational forces[10]. Visual information, specifically the orientation of our environment and affordances[13] from objects in the environment, provide directional cues associated with gravity, enhancing one's perception of internal orientation and defining their position in space[8]. Previous studies have shown that sensorimotor adaptation and afferent signal reweighting during space flight manifests itself as changes to static balance stability[12] and dynamic stability during locomotion[10,14,15]. Gaze stabilization and dynamic visual acuity changes are also observed, and have been associated with vestibular changes in the vestibular-ocular reflex (VOR)[16] and locomotor head-trunk coordination[14,15,17] upon return to Earth.

The Functional Task Test (FTT) is a set of seven functional activities used to assess astronaut 
performance that is coupled to a set of interdisciplinary physiological measurements to identify factors which contribute to functional decrements, for use in countermeasure design. Some of these FTT activities and their focuses include: seat egress and walk (coordinated movement time), recovery from fall-tostand (postural stability, orthostatic intolerance), open/close a hatch (torque generation) and ladder climb. The seat egress and walk task, the focus of this study, requires musculoskeletal strength and balance, as well as coordinated head-and-trunk movement to successfully navigate an obstacle course, and is measured in time to complete the course. During the slalom portion of the obstacle course, participants are required to make three turns (approximately $60^{\circ}$ ) around upright pillars as quickly as possible, without running or colliding with the obstacles. The quantification of whole body turns while walking around corners (changing direction by $90^{\circ}$ ) show the systemic movement of the head and eyes towards the new trajectory, followed by the torso and feet during steering locomotion[18,19]. Hollands et al.[20] extended this research isolating the relationship between head and trunk turning in increments of $30^{\circ}$ and $60^{\circ}$ during normal steering and trajectory planning, as well as an experimentally controlled head-to-trunk locked body posture. These authors found that when natural head-to-trunk movement was compromised, both control of trunk reorientation and control of body center of mass (COM) translation were significantly affected.

Head-to-trunk locking, or 'en bloc' movement of the head and trunk during locomotion, has been shown to relate in adulthood to ambulation in an increasingly difficult environment (unfamiliar or unstable)[21], or to systemic impairments as a result of pathology, such as vestibular impairment[15] or Parkinson's disease[22,23]. Individuals experiencing chronic vestibular loss (bilateral labyrinth deficiencies) have also been shown to reduce their head pitch amplitude and adopt a head-trunk locking strategy when compared to normal subjects[15]. Various mechanisms have been described to contribute to changes in head trunk coordination strategies, such as a gaze focused feedforward system[24], a feed-back systems grounded in the vestibular-ocular reflex (VOR) and vestribulo-collic reflex (VCO)[25], or simply a vestibular input mediated response to provide head stabilization in space[26]. Parkinsonians, specifically, show the appropriate control strategy, however demonstrate decreased headto-trunk movement latencies as well as decreased head and trunk movement velocity during walking turns. Akram et al.[27] also showed increased 'en bloc' movement in elderly populations during walking $90^{\circ}$ turns when visual information was limited, such as an eyes-closed condition. Evidence of the influence of microgravity on head-trunk coordination has been shown as an increase in head-pitch-to-trunk locking while treadmill walking during a gaze stabilization task, upon return to Earth[14,15,17].

To better understand how microgravity impacts physiology and performance, six-degree head down tilt (HDT) bed rest campaigns have been used as a ground based analog to space flight, and have been shown to successfully reproduce muscular and cardiovascular deconditioning[28,29]. In a corresponding study (CFT70), participants were tested using the FTT protocol before and after 70 days of $6^{\circ}$ head-down bedrest as an analog for space flight. Bed-rest provides the opportunity to investigate the role of prolonged axial body unloading in isolation from the other physiological effects produced by exposure to the microgravity environment of space flight. In this way, bed rest allows us to investigate the impact of body unloading on both functional, mission related, tasks and on the underlying physiological changes that lead to decrements in performance, allowing comparison with results obtained during space flight studies

The purpose of this study was to investigate the effects of microgravity exposure and 70-day HDT bed rest unloading on the head-trunk coordination of participants during over-ground locomotion through an obstacle course. We hypothesized that, given the potential influence of the vestibular system in such a control strategy, long duration exposure to microgravity would result in a profound neuro-vestibular adaptation exhibited by head-to-trunk locking during walking turns and obstacle avoidance. This 'en bloc' movement would be characterized by a decrease in the latency between head and trunk movements. Long duration bed-rest participants, however, would not experience a shift in their head-to-trunk locking strategy, due to their consistent exposure to Earth's gravitational vector. Decrements to head-trunk coordination during ambulation in astronauts returning to a gravity environment represents a deviation from the nominal movement strategy and may pose a risk to astronaut safety, especially in an unfamiliar, low visibility or unstable environment. 


\section{METHODS}

\section{II.I Subjects}

Data collected from thirteen astronauts who participated in long-duration missions (6 months) aboard the International Space Station (ISS) are presented in this study. This sample includes 13 astronaut participants. Participants performed the Functional Task Test (FTT) battery near or at 30 (L-30), 60 (L-60) and 180 (L-180) days before launch, as well as $1(\mathrm{R}+1), 6(\mathrm{R}+6)$ and 30 $(\mathrm{R}+30)$ days after return from space flight. This test battery included seven functional tests, designed to mimic functional activities performed by astronauts during the course of a mission. A subset of collected crewmember data is presented in this study, including one pre-flight (L-30) and one post-flight $(\mathrm{R}+1)$ measurement to highlight acute changes due to microgravity exposure. All subjects gave informed consent according to the requirements of the Institutional Review Board at NASA Johnson Space Center.

Data collected from 26 subjects who participated in a 70-day 6 degree head down tilt (HDT) bed-rest cohort are presented as a ground-based space flight analog in this study. This sample included 26 male participants, 16 of which were assigned to an exercise group (BRE) and 10 who were control subjects (BRC), participating in no exercise during their bed-rest. BRE participants performed resistance, interval aerobic and continuous aerobic exercises while maintaining 6 degree HDT on specially designed exercise equipment, such as a horizontal squat machine, a horizontal cycle ergometer and horizontal treadmill. Participants performed the same Functional Task Test battery 1 (BR-1), 7 (BR-7) and 12 (BR-12) days before entering bed-rest and 0 (immediately out of bed, R+0), $1(\mathrm{R}+1), 6(\mathrm{R}+6)$ and 12 $(\mathrm{R}+12)$ days following the completion of the bed-rest protocol. A subset of collected bed-rest data is presented in this study, including one pre-bed-rest (BR1) and one post-bed-rest $(\mathrm{BR}+0)$ measurement to highlight acute changes due to bed-rest deconditioning. All subjects gave informed consent according to the requirements of the Institutional Review Board at NASA Johnson Space Center.

\section{II.II Data Collection}

The Seat Egress and Walk Test, a component of the FTT (Figure 1), involved the unbuckling of a four-point harness from a seated position, the completion of an obstacle course including an over-under 'portal', followed by a four pillar slalom and an elevated ramp, at the end of which a button was pressed. A final trigger was activated when the participant returned down the ramp to complete the trial.



Fig. 1: The Seat Egress and Walk Test, one of seven functional activities used to assess performance after exposure to unloading, either microgravity or bed rest.

Participants were instructed to complete the obstacle course as quickly as possible, without running, while minimizing interaction with the obstacles. Head and trunk movement kinematics were recorded using XSens MTx 6 degree-of-freedom inertial measurement units (XSens Technologies B.V., Enschede, Netherlands) attached to the head and upper back, and were sampled at $50 \mathrm{~Hz}$. For the purposes of this study, only data collected during the slalom portion of the obstacle course is presented. Pillars in this segment were equally spaced, staggered out of phase, approximately $45^{\circ}$ from one another, spanning a distance of approximately $4 \mathrm{~m}$. Navigation of this slalom portion results in three turns (approximately $60^{\circ}$ each). Light-gates and pressure switches were used to produce trigger events to accurately identify the initiation and completion of the slalom section of the obstacle course.

\section{II.III Data Analysis}

The kinematic data were processed off-line using custom Matlab scripts (Mathworks Inc., Massachusetts, USA). For the purposes of this analysis, only yaw rotation data, measured in degrees, was observed to describe movement of the head and torso about the long axis of the body (Z-axis). Rotation data was low pass filtered at $5 \mathrm{~Hz}$ using a zero-lag $2^{\text {nd }}$ order Butterworth filter. A $5 \mathrm{~Hz}$ low-pass filtering frequency was selected in accordance with Nyquist frequency theory[30], such that the filtering frequency was at least twice that of the peak frequency bandwidth. Two minimum and maximum rotational extrema were identified to describe the turning trajectories during obstacle avoidance, for 
both the head and trunk mounted IMUs (Figure 2). Head-to-trunk yaw latency was used as a metric to characterize changes in head trunk coordination. Headto-trunk turning latency $(\tau)$ is defined as the absolute value of the average time delay (sinusoidal phase shift) between head and trunk extrema (Eq. 1)

$$
\begin{aligned}
& \tau_{\text {latency }}= \\
& \left|\frac{\sum_{1}^{i}\left(t_{\operatorname{maxi}\left(\theta_{h}\right)_{i}}-t_{\operatorname{maxi}\left(\theta_{t}\right)_{i}}\right)+\sum_{1}^{j}\left(t_{\operatorname{mini}\left(\theta_{h}\right)_{j}}-t_{\left.\operatorname{mini}\left(\theta_{t}\right)_{j}\right)}\right.}{i+j}\right|
\end{aligned}
$$

where $t_{\text {maxi }}$ and $t_{\text {mini }}$ represent the time at which maxima $(i)$ or minima $(j)$ were observed for either the rotation of the head $\left(\Theta_{h}\right)$ or the torso $\left(\Theta_{t}\right)$ observed during the slalom section. Figure 2 shows a sample of maxima and minima differences identified to determine head-to-trunk movement latency. A decrease in headto-trunk movement latency represented a shift towards head-and-trunk locked, or 'en bloc' movement, while an increase in latency describeed a shift to more independent head-and-trunk movement.

Head-to-trunk latency was compared between L-30 and $\mathrm{R}+1$ in space flight participants (Figure 2), as well as BR-1 and $\mathrm{BR}+0$ in bed rest participants, for both control and exercise groups. All participants were grouped as 'decreaser' or 'increaser,' relating to their change in head-to-trunk movement latency between pre- and post- environmental adaptation measures.

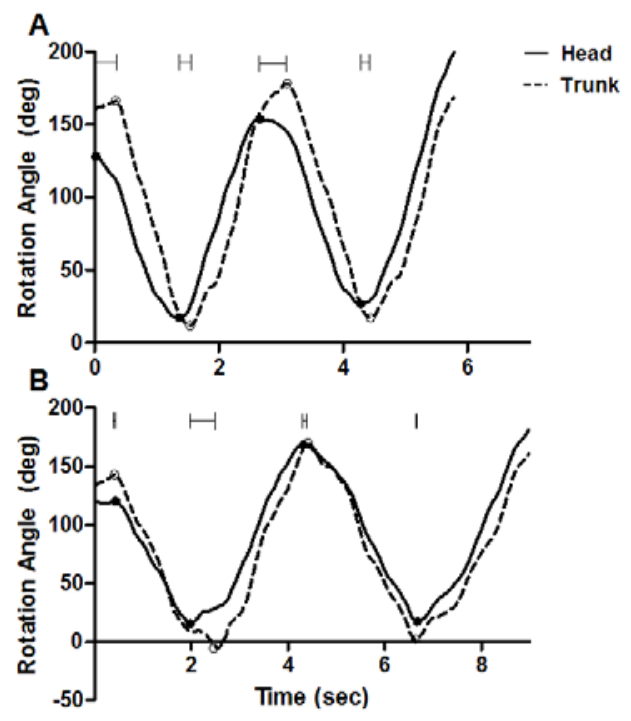

Fig. 2: A: Head and trunk yaw rotation angles for slalom section of obstacle course with maxima and minima differences highlighted in a single Pre-flight (L-30) astronaut subject. B: Post-flight $(\mathrm{R}+1)$ results for the same subject showing decreased latency between head-and-trunk movement.

\section{II.IV Statistics}

For the space flight analysis and bed rest participants (control and exercise groups), subject's data collected prior to unloading ('pre-' flight or bed rest) was compared to their corresponding data collected after unloading ('post-' flight or bed rest). Paired t-tests were performed for each group (ISS, BRE and BRC) to characterize the average adaptation change in headtrunk latency. Statistical significance was defined at alpha $<0.05$ for all tests.

\section{$\underline{\text { III. RESULTS }}$}

Participants in each group (ISS, BRE and BRC) were further stratified based on their performance during the Seat Egress and Walk Test: those who experienced an increase in head-to-trunk latency after exposure to unloading ('increaser') and those who showed a decrease in head-to-trunk latency (or increased head-totrunk locking) during movement ('decreaser').

\section{III.I Space Flight Participants (ISS)}

Astronauts who experienced six months of microgravity exposure aboard the International Space Station showed a bimodal response in head-trunk coordination, where 6 out of 13 (46.2\%) of participants were labelled "increaser," showing an increased latency in head-totrunk movement during the slalom movement. The rest (7 of 13 , or $53.8 \%$ of participants) showed an increase in head-to-trunk locking ('decreaser' in latency) during $60^{\circ}$ turns in the Seat Egress and Walk test (Figure 3). Both the increaser $(\mathrm{t}(6)=9.16, \mathrm{p}<0.05)$ and decreaser $(\mathrm{t}(5)=6.24, \mathrm{p}<0.05)$ groups experienced significant changes in head-to-trunk coordination between pre- and post- microgravity exposure measurements. 


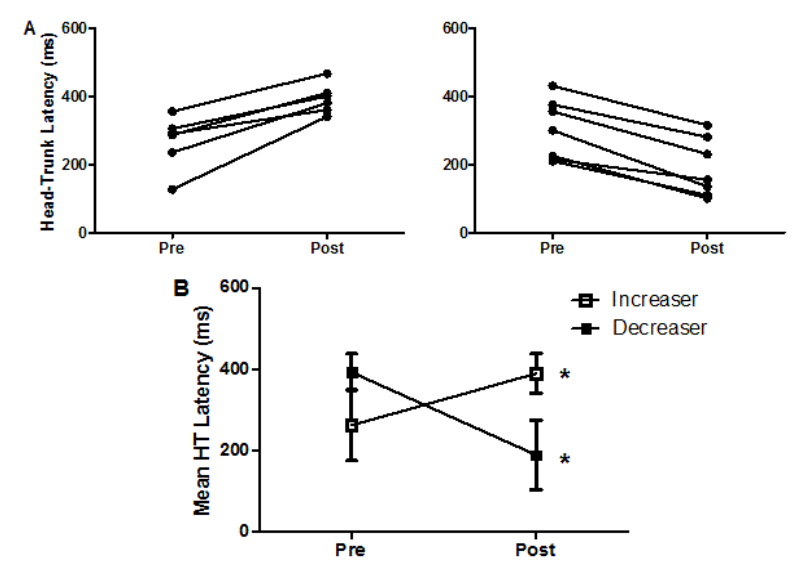

Fig. 3: A: Individual responses pre- and post- 6 month microgravity (ISS) exposure highlighting bimodal 'increaser' and 'decreaser' response. B: Statistically significant mean differences in both groups after long duration space flight.

\section{III.II Bed Rest Control Group (BRC)}

Bed rest participants who did not take part in aerobic or resistive exercise during their 70-day head down bed rest unloading exposure (bed rest controls) appeared to favor an increased head-to-trunk locking strategy while navigating obstacles in the slalom portion of the obstacle course (Figure 4). Of the 10 BRC participants, $80 \%$ showed significantly decreased head-to-trunk latency $(t(7)=4.29, p<0.05$ ), while the final two showed a minimal increase in head-to-trunk latency, which was not significantly different between pre- and post- bed rest measurements $(t(1)=-2.31, \mathrm{p}>0.05)$.

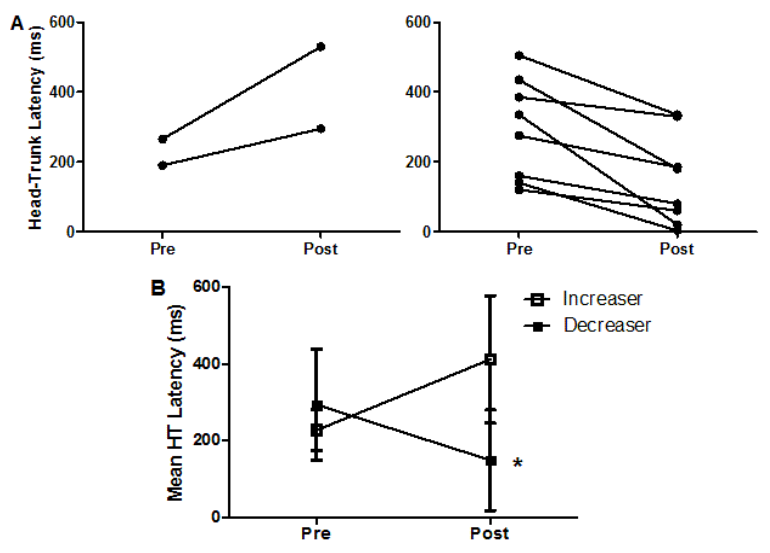

Fig. 4: A: Individual responses pre- and post- 70-day head down tilt bed rest for controls (BRC) receiving no exercise. B: Significantly different response in 'decreaser' group when compared to baseline measures.

\section{III.III Bed Rest Exercise Group (BRE)}

Bed rest participants who adhered to a strict regimen of high and low intensity aerobic, as well as resistance, exercise showed similar responses to those who took part in no exercise. BRE participants also tended to favor an increased head-to-trunk locking strategy during the Seat Egress and Walk test (Figure 5). Of the 16 BRE participants, $81.3 \%$ showed significantly decreased head-to-trunk latency (identified as "decreasers", $t(12)=6.16, p<0.05)$, while the remaining 3 participants (identified as "increasers") showed no significant differences between their pre- and post- bed rest measurements $(\mathrm{t}(2)=-1.44, \mathrm{p}>0.05)$.

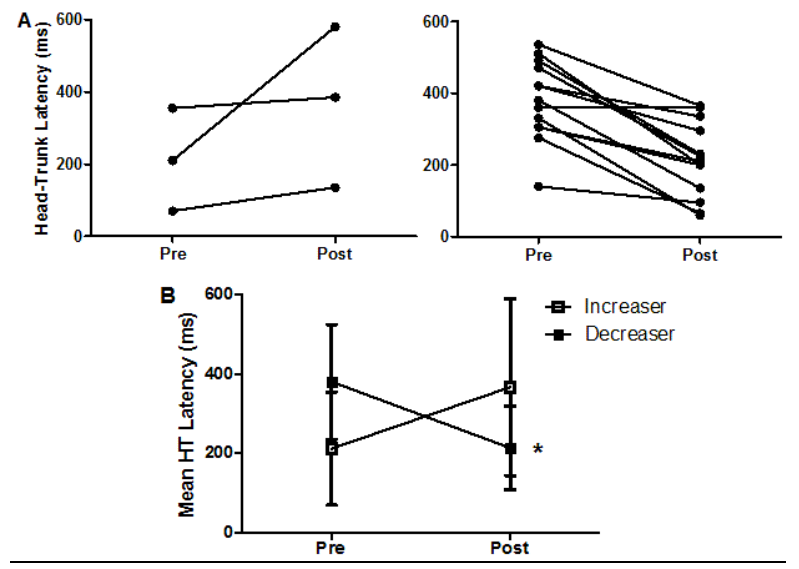

Fig. 5: A: Individual responses pre- and post- 70-day head down tilt bed rest for those who exercised regularly. B: Significantly different response in 'decreaser' group when compared to baseline measures.

\section{DISCUSSION}

The purpose of this study was to identify changes in head-to-trunk coordination after exposure to unloading via long duration space flight (microgravity exposure aboard the International Space Station) or long duration $6^{\circ}$ head-down bed rest. Kinematic data of head and trunk movement during the Seat Egress and Walk Test were collected in all groups of participants pre- and post- unloading. Using the yaw rotation data collected about the long axis of the body ( $\mathrm{Z}$ axis), the latency (msec) between head and trunk movement $(\tau)$ was calculated while traversing the slalom portion of the Seat Egress and Walk Test (approximately four $60^{\circ}$ turns). Results showed varying responses between participants in all groups, where some were observed to increase the latency between head and trunk movement ('increaser' group), while others decreased their movement latency ('decreaser' group), adopting a head- 
locking, or 'en bloc,' movement strategy. Space flight unloading (ISS) showed the greatest divergence between the 'increaser' and 'decreaser' group, while both bed rest control (BRC) and bed rest exercise (BRE) populations showed increased preference towards a 'decreaser' categorization, displaying greater head-trunk locking.

IV.I Effects of space flight on head-trunk coordination during ambulation and obstacle avoidance

A bimodal relationship was observed during stratification of astronaut participants into groups of 'increaser' or 'decreaser' latency, such that the population was split almost evenly. Each group also exhibited significant changes in latency during wholebody turns: 'increasers' showed more head-to-trunk movement, while 'decreasers' showed more head-totrunk locking. It is interesting to observe two distinct responses, both significantly changed from baseline values. These results reinforce previous findings observing changes in head-and-trunk coordination (pitch plane) after microgravity exposure[15,17], where participants were also observed to display a diverse response of 'increasers' and 'decreasers.' We interpret these results to suggest an individualized strategy of sensorimotor reweighting during the transitory period upon return to Earth. Individuals who adopt a strategy of down-regulating vestibular afferent feedback during microgravity, depending primarily on visual information for spatial orientation, may be more susceptible to vestibular inputs upon return to Earth. This response explains the increase in head-locking of the 'decreaser' group, as they attempt to minimize vestibular inputs. Although head rotations observed in this study are primarily characterized by semicircular canal (rotational) afferent information, the movement vectors imposed by ambulation likely impart otolith stimulation as well. Investigation of the adaptation of vestibular organs to microgravity in animal models have shown marked increases in nerve afferents from otolith organs in toadfish[31], resulting in enhanced sensitivity. Individuals who adopt a strategy of increased head-trunk latency ('increaser' group) may have a greater dependency on vestibular inputs, making them more susceptible to uncontrolled movement during the microgravity to $1 \mathrm{~g}$ transition. Individualized and varied adaptation strategies have become a reoccurring theme in recent space flight research, observing the influence of visual versus somatosensory dominance during sensorimotor integration for spatial orientation and motor performance[32,33].
A distinct limitation in this population includes the fact that astronauts are tested after 24-36 hours of exposure to Earth gravity. This delay represents the time between re-entry, occurring in Russia, and testing at the Johnson Space Center in Houston, Texas. Sensorimotor readaptation to Earth gravity is expected to begin as soon as one returns to the surface of the Earth, and provides a presently unavoidable confounding factor to our observations. Individuals who are rapid or experienced re-adaptors, due to flexibility or generalizability of their motor learning systems[34] may show inherently improved performance on functional tests compared to slower adaptors.

IV.II Results of prolonged axial body unloading in head down bed rest on head-trunk coordination

Six degree head down tilt bed rest campaigns have been used for many years as an analog to simulate the deleterious effects of microgravity exposure[29], and remains a consistently used protocol for better understanding and mitigating these physiological problems. Bed rest traditionally provides a good representative model of cardiovascular and musculoskeletal deconditioning, and recently has been determined to serve as an exclusionary analog[35] to better observe changes unique to body unloading and associated changes in the vestibulo-spinal system. In groups of bed rest participants who exercised (BRE) and those who were experimental controls (BRC), a predominant shift towards a decrease in head-to-trunk latency ('en bloc' movement) was observed when comparing baseline measures to ambulation immediately upon exiting bed rest $(\mathrm{BR}+0)$. Individuals experiencing bed rest, unlike those on the ISS, consistently receive the Earth's $1 \mathrm{~g}$ of loading throughout their body; however due to their reorientation, they are not receiving the axial somatosensory body loading we, as humans, have evolved to expect. Participants newly out of bed rest often remark of plantar discomfort or soreness, primarily due to disuse. This skin sensitivity resembles changes observed in short-duration space flight participants and may contribute to changes in movement strategy[36]. It is possible, however, that due to abnormal and inconsistent somatosensory unloading, despite periodic bouts of exercise and loading in some individuals, vestibular adaptations may be have occurred.

Recent evidence supporting vestibular-somatosensory convergence through the observation of vestibularly mediated head movement control[15] has further 
confirmed this mechanism. Healthy, body unloaded, individuals, returning astronauts and individuals with bilateral vestibular deficiencies were observed to produce adaptive changes in head movement control during treadmill locomotion. In this study, angular head pitch and trunk vertical translation movement were recorded during treadmill walking while performing a gaze stabilization task. Adaptation to whole body unloading while walking caused a significant increase in head pitch movements in normal participants.. In contrast, subjects with bilateral vestibular deficits showed a decrease in compensatory pitch head movements. Astronauts measured in this way showed the aforementioned bimodal response: some increased in head pitch, like the body unloaded subjects, while others decreased, like subjects with vestibular deficits[15]. Previous work in animals by Jian et al.[37] have shown that non-labyrinthine inputs from the limbs of cats modulate signals from the vestibular nuclei, and suggest that it is likely that a similar mechanism exists in all mammals. A corresponding study of Russian cosmonauts, observing the influence of support afferentation by providing foot pressure during space flight, provides further support for vestibular modulation via somatosensory stimuli. Cosmonauts who received support afferentation demonstrated improved eye-head coordination performance over those who perform only vestibular countermeasures (active head rotations) and those who performed no countermeasures (free floating)[38]. Finally, recent neuro imaging studies have shown the vestibular system to be multisensory in nature, where vestibular stimulation activates somatosensory areas in the brains of primates. Specific evoked potentials (N80) were observed under experimental conditions, suggesting that vestibular stimulation modulates somatosensory cortical processing[39]. These results provide preliminary mechanistic evidence supporting an interaction between vestibular and somatosensory systems, required for vestibular-somatosensory convergence during sensorimotor integration.

The goal of this study was to identify changes in headto-trunk coordination during ambulation of an obstacle course after exposure to long duration body weight unloading. Results obtained from astronauts who participated in 6 months of microgravity exposure aboard the International Space Station showed a bimodal response to head-trunk coordination changes. Some experienced increases in head-to-trunk movement latency, while others experienced a decrease in latency, also known as 'en bloc' movement of the head-and- trunk, during obstacle avoidance. Individualized responses to microgravity adaptations have been observed in other measures of head-trunk coordination and gaze stabilization [15-17], and are consistent in this study. Results obtained from participants who experienced 70 days of head down tilt bed rest showed a predominant shift towards head-to-trunk locking strategies during ambulation of the same obstacle course. These results were mirrored in both the control and exercise groups of bed rest participants, suggesting no effects of exercise on head-trunk coordination after bed rest unloading. These results provide further support for the concept of vestibular-somatosensory convergence, such that extended body weight unloading with minimized afferent proprioceptive feedback results in changes to vestibular-somatosensory integration in the sensorimotor cortex. These results provide key insights for the future of individualized training programs and microgravity countermeasures, supporting the notion that only a set of integrated countermeasures - the simultaneous stressing multiple physiological systems - will be successful in mitigating the effects of microgravity unloading during space flight.

\section{REFERENCES}

[1] T. Lang, A. LeBlanc, H. Evans, Y. Lu, H. Genant, A. Yu, Cortical and Trabecular Bone Mineral Loss From the Spine and Hip in LongDuration Spaceflight, J. Bone Miner. Res. 19 (2004) 1006-1012. doi:10.1359/JBMR.040307.

[2] A.D. LeBlanc, E.R. Spector, H.J. Evans, J.D. Sibonga, Skeletal responses to space flight and the bed rest analog: a review, J. Musculoskelet. Neuronal Interact. 7 (2007) 33.

[3] J. Rittweger, H. Frost, H. Schiessl, H. Ohshima, B. Alkner, P. Tesch, et al., Muscle atrophy and bone loss after 90 days' bed rest and the effects of flywheel resistive exercise and pamidronate: Results from the LTBR study, Bone. 36 (2005) 1019-1029. doi:10.1016/j.bone.2004.11.014.

[4] V.A. Convertino, Status of cardiovascular issues related to space flight: Implications for future research directions, Respir. Physiol. Neurobiol. 169 (2009) S34-S37. doi:10.1016/j.resp.2009.04.010.

[5] V.J. Caiozzo, F. Haddad, S. Lee, M. Baker, W. Paloski, K.M. Baldwin, Artificial gravity as a countermeasure to microgravity: a pilot study examining the effects on knee extensor and plantar flexor muscle groups, J. Appl. Physiol. 107 (2009) 39-46. doi:10.1152/japplphysiol.91130.2008. 
$65^{\text {th }}$ International Astronautical Congress, Toronto, Canada. Copyright (C2014 by the International Astronautical Federation. All rights reserved.

[6] A.D.J. Moore, S.M. Lee, J.B. Charles, M.C. Greenisen, S.M. Schneider, Maximal exercise as a countermeasure to orthostatic intolerance after spaceflight., Med. Sci. Sports Exerc. 33 (2001).

[7] B.J. Yates, I.A. Kerman, Post-spaceflight orthostatic intolerance: possible relationship to microgravity-induced plasticity in the vestibular system, Brain Res. Rev. 28 (1998) 73-82.

[8] G. Clément, J.T. Ngo-Anh, Space physiology II: adaptation of the central nervous system to space flight-past, current, and future studies, Eur. J. Appl. Physiol. 113 (2013) 1655-1672. doi:10.1007/s00421-012-2509-3.

[9] S.J. Wood, J.A. Loehr, M.E. Guilliams, Sensorimotor reconditioning during and after spaceflight, NeuroRehabilitation. 29 (2011) 185195.

[10] M.F. Reschke, J.J. Bloomberg, D.L. Harm, W.H. Paloski, C. Layne, V. McDonald, Posture, locomotion, spatial orientation, and motion sickness as a function of space flight, Brain Res. Rev. 28 (1998) 102-117.

[11] W.H. Paloski, Vestibulospinal adaptation to microgravity, Otolaryngol. Neck Surg. 118 (1998) s39-s44.

[12] R.A. Speers, W.H. Paloski, A.D. Kuo, Multivariate changes in coordination of postural control following spaceflight, J. Biomech. 31 (1998) 883-889.

[13] J.J. Gibson, The Theory of Affordances, in: Ecol. Approach Vis. Percept., Houghton Mifflin, Boston, 1979: pp. 127-143.

[14] J.J. Bloomberg, A.P. Mulavara, Changes in walking strategies after spaceflight, Eng. Med. Biol. Mag. IEEE. 22 (2003) 58-62.

[15] A.P. Mulavara, T. Ruttley, H.S. Cohen, B.T. Peters, C. Miller, R. Brady, et al., Vestibularsomatosensory convergence in head movement control during locomotion after long-duration space flight, J. Vestib. Res. 22 (2012) 153-166.

[16] B.T. Peters, C.A. Miller, R.A. Brady, J.T. Richards, A.P. Mulavara, J.J. Bloomberg, Dynamic Visual Acuity During Walking After Long-Duration Spaceflight, Aviat. Space Environ. Med. 82 (2011) 463-466. doi:10.3357/ASEM.2928.2011.

[17] J.J. Bloomberg, B.T. Peters, S.L. Smith, W.P. Huebner, M.F. Reschke, Locomotor head-trunk coordination strategies following space flight., J. Vestib. Res. Equilib. Orientat. 7 (1997) 161-177.

[18] R. Grasso, P. Prévost, Y.P. Ivanenko, A. Berthoz, Eye-head coordination for the steering of locomotion in humans: an anticipatory synergy, Neurosci. Lett. 253 (1998) 115-118.

[19] D. Solomon, Vijay Kumar, R.A. Jenkins, J. Jewell, Head control strategies during whole- body turns, Exp. Brain Res. 173 (2006) 475-486. doi:10.1007/s00221-006-0393-y.

[20] M. Hollands, K. Sorensen, A. Patla, Effects of head immobilization on the coordination and control of head and body reorientation and translation during steering, Exp. Brain Res. 140 (2001) 223-233. doi:10.1007/s002210100811.

[21] S. Nadeau, B. Amblard, S. Mesure, D. Bourbonnais, Head and trunk stabilization strategies during forward and backward walking in healthy adults, Gait Posture. 18 (2003) 134142.

[22] F. Huxham, R. Baker, M.E. Morris, R. Iansek, Head and trunk rotation during walking turns in Parkinson’s disease, Mov. Disord. 23 (2008) 1391-1397. doi:10.1002/mds.21943.

[23] S. Akram, J.S. Frank, M. Jog, Parkinson’s disease and segmental coordination during turning: II. Walking turns., Can. J. Neurol. Sci. J. Can. Sci. Neurol. 40 (2013) 520-526.

[24] M.A. Hollands, N.V. Ziavra, A.M. Bronstein, A new paradigm to investigate the roles of head and eye movements in the coordination of wholebody movements, Exp. Brain Res. 154 (2004) 261-266. doi:10.1007/s00221-003-1718-8.

[25] M.F. Land, The coordination of rotations of the eyes, head and trunk in saccadic turns produced in natural situations, Exp. Brain Res. 159 (2004) 151-160. doi:10.1007/s00221-004-1951-9.

[26] C. Assaiante, B. Amblard, Ontogenesis of head stabilization in space during locomotion in children: influence of visual cues, Exp. Brain Res. 93 (1993) 499-515.

[27] S.B. Akram, J.S. Frank, J. Fraser, Coordination of segments reorientation during on-the-spot turns in healthy older adults in eyes-open and eyes-closed conditions, Gait Posture. 32 (2010) 632-636. doi:10.1016/j.gaitpost.2010.09.006.

[28] G.R. Adams, V.J. Caiozzo, K.M. Baldwin, Skeletal muscle unweighting: spaceflight and ground-based models., J. Appl. Physiol. Bethesda Md 1985. 95 (2003) 2185-2201. doi:10.1152/japplphysiol.00346.2003.

[29] A. Pavy-Le Traon, M. Heer, M.V. Narici, J. Rittweger, J. Vernikos, From space to Earth: advances in human physiology from 20 years of bed rest studies (1986-2006), Eur. J. Appl. Physiol. 101 (2007) 143-194. doi:10.1007/s00421-007-0474-z.

[30] H. Nyquist, Certain topics in telegraph transmission theory, Proc. IEEE. 90 (2002) 280305.

[31] R. Boyle, A.F. Mensinger, K. Yoshida, S. Usui, A. Intravaia, T. Tricas, et al., Neural readaptation to Earth's gravity following return from space, J. Neurophysiol. 86 (2001) 2118-2122. 
$65^{\text {th }}$ International Astronautical Congress, Toronto, Canada. Copyright (C2014 by the International Astronautical Federation. All rights reserved.

[32] R.A. Brady, B.T. Peters, C.D. Batson, R. PloutzSnyder, A.P. Mulavara, J.J. Bloomberg, Gait adaptability training is affected by visual dependency, Exp. Brain Res. 220 (2012) 1-9. doi:10.1007/s00221-012-3109-5.

[33] C.D. Batson, R.A. Brady, B.T. Peters, R.J. Ploutz-Snyder, A.P. Mulavara, H.S. Cohen, et al., Gait training improves performance in healthy adults exposed to novel sensory discordant conditions, Exp. Brain Res. 209 (2011) 515-524. doi:10.1007/s00221-011-2574-6.

[34] R.D. Seidler, Multiple motor learning experiences enhance motor adaptability, J. Cogn. Neurosci. 16 (2004) 65-73.

[35] M.F. Reschke, J.J. Bloomberg, W.H. Paloski, A.P. Mulavara, A.H. Feiveson, D.L. Harm, Postural Reflexes, Balance Control, and Functional Mobility with Long-Duration HeadDown Bed Rest, Aviat. Space Environ. Med. 80 (2009) 45-54. doi:10.3357/ASEM.BR06.2009.

[36] C.R. Lowrey, S.D. Perry, N.D.J. Strzalkowski, D.R. Williams, S.J. Wood, L.R. Bent, Selective skin sensitivity changes and sensory reweighting following short-duration space flight, J. Appl. Physiol. 116 (2014) 683-692. doi:10.1152/japplphysiol.01200.2013.

[37] B.J. Jian, T. Shintani, B.A. Emanuel, B.J. Yates, Convergence of limb, visceral, and vertical semicircular canal or otolith inputs onto vestibular nucleus neurons, Exp. Brain Res. 144 (2002) 247-257. doi:10.1007/s00221-002-10428.

[38] L. Kornilova, V. Grigorova, C. Mueller, S.V. Sagalovitch, M. Alekhina, I. Kozlovskaya, Effects of vestibular and support afferentation upon visual pursuit in microgravity., J. Gravitational Physiol. J. Int. Soc. Gravitational Physiol. 11 (2004).

[39] E.R. Ferrè, G. Bottini, P. Haggard, Vestibular inputs modulate somatosensory cortical processing, Brain Struct. Funct. 217 (2012) 859864. doi:10.1007/s00429-012-0404-7. 